\title{
Selective phonotaxis of female crickets under natural outdoor conditions
}

\author{
Stefan Hirtenlehner $\cdot$ Heiner Römer
}

Received: 15 November 2013 / Revised: 7 January 2014 / Accepted: 10 January 2014 / Published online: 1 February 2014

(C) The Author(s) 2014. This article is published with open access at Springerlink.com

\begin{abstract}
Acoustic mate choice in insects has been extensively studied under laboratory conditions, using different behavioural paradigms. Ideally, however, mate choice designs should reflect natural conditions, including the physical properties of the transmission channel for the signal. Since little is known about the discrimination ability of females between male song variants under natural conditions, we performed phonotaxis experiments with female field crickets (Gryllus bimaculatus) outdoors, using two-choice decisions based on differences in carrier frequency, sound pressure level, and chirp rate. For all three song parameters, minimum differences necessary for a significant preference between two song models were considerably larger outdoors compared to laboratory conditions. A minimum amplitude difference of $5 \mathrm{~dB}$ was required for a significant choice in the field, compared to only $1-2 \mathrm{~dB}$ reported for lab-based experiments. Due to the tuned receiver system, differences in carrier frequency equal differences in perceived loudness, and the results on choice for differences in carrier frequency corroborate those in amplitude. Similarly, chirp rate differences of $50 \mathrm{chirps} / \mathrm{min}$ were required outdoors compared to only $20 \mathrm{chirps} / \mathrm{min}$ in the lab. For predictions about patterns of sexual selection, future studies need to consider the different outcomes of mate choice decisions in lab and field trials.
\end{abstract}

Keywords Phonotaxis $\cdot$ Female choice $\cdot$ Acoustic communication $\cdot$ Field cricket $\cdot$ Decision making

S. Hirtenlehner $(\bowtie) \cdot$ H. Römer

Department of Zoology, Karl-Franzens-University,

Universitätsplatz 2, $8010 \mathrm{Graz}$, Austria

e-mail: stefan.hirtenlehner@uni-graz.at

\author{
Abbreviations \\ CF Carrier frequency \\ GLMM Generalized linear mixed model \\ IID Interaural intensity difference \\ JMD Just meaningful difference \\ JND Just noticeable difference \\ SPL Sound pressure level
}

\section{Introduction}

Numerous studies with taxa from invertebrates to mammals have documented that males with certain attributes gain a mating advantage through female mate choice (Andersson 1994). Acoustic mate choice has been extensively studied in the past, since it offers several advantages compared to other sensory modalities. The signals being involved can easily be recorded in the field and laboratory, and the acoustic behaviour is reliably elicited in response to playbacks, in which all signal parameters are under precise control of the experimenter using audio software processing. In this way, the selectivity of receivers for certain acoustic parameters has been determined for different species, providing a framework for studying mechanisms underlying species recognition and for generating predictions about patterns of sexual selection and speciation (Gerhardt and Huber 2002; Bradbury and Vehrencamp 2011).

In particular, insects are excellent model systems to study decisions based on acoustic signals (review Gerhardt and Huber 2002; Hedwig 2006). In field crickets, for example, positive phonotaxis of the female towards the calling song of the male is the first step in mate choice. During courtship and (potentially following) copulation females may further assess other cues, such as cuticular hydrocarbons, which can provide information important for species 
and kin recognition (Alexander 1962; Singer 1998; Wagner and Reiser 2000; Thomas and Simmons 2009; Simmons et al. 2013).

The calling song is produced by male crickets with a species-specific temporal pattern (Otte 1992; Pollack 1998). Besides field studies in which the attraction of females towards males with certain call parameters or body traits has been quantified, or even their mating success recorded (Ulagaraj and Walker 1975; Walker and Forrest 1989; Farris et al. 1998; Rodríguez-Muñoz et al. 2010), the vast majority of studies was laboratory based, and used either trackballs, walking compensators or arenas to quantify the behaviour of receivers (Gerhardt and Huber 2002 for review). Each of these approaches has its special advantages and limitations due to the freedom of movement of the females, the preciseness of control over stimulus parameters, or whether females perform phonotaxis under open- or closed-loop conditions.

However, the ability of receivers to detect and discriminate the differences in male traits which are used for their mating decisions are also influenced by environmental conditions. Although the acoustic communication channel bears some advantages over the visual or olfactory channel, acoustic signals may be strongly degraded and attenuated by physical properties of the environment. A potentially informative character, for example, the interval between sound pulses, is degraded by reflection or echoes (Wiley and Richards 1978; Römer and Lewald 1992; Römer 1998, 2001). Furthermore, background noise can result in masking of the signal or signal components (Brumm and Slabbekoorn 2005; Römer 2013). These conditions suggest that a signal that functions efficiently in the laboratory, and can be easily discriminated from another signal variant, may not be ideal in the natural environment where individuals communicate (Endler 1992). This may explain why in several frog species the preferences of females for differences in carrier frequency or intensity as observed in the laboratory are not reflected in outdoor settings (Gerhardt 1992; Dyson et al. 1992).

Although female mate choice in insects has been studied extensively in the laboratory, phonotaxis has only rarely been observed and quantified in the natural habitat (but see Mhatre and Balakrishnan 2008 for an exception with a cricket species; and Hirtenlehner et al. 2014 for a no-choice situation). Indeed, in a field study on a wild population of Teleogryllus commodus Bentsen et al. (2006) showed a strong stabilizing sexual selection on call structure verifying results previously found under laboratory conditions (Brooks et al. 2005). Outdoor conditions are particularly challenging for female cricket receivers, and the experimenter recording and quantifying the behaviour, for several reasons. One is the problem of sound transmission parallel to the ground which can create complicated patterns of interactions between the direct wave and the one reflected from the ground (reviewed in Embleton 1996; see also Römer 2001). Indeed, in a recent outdoor study using neurophysiological methods it has been shown that both directional and distance information can be strongly degraded for a receiver at some distance from the source (Kostarakos and Römer 2010). Furthermore, in the grassland the female is not completely free to orient straight towards a sound source due to obstacles in the transmission channel, so that forced turns might bias the walking direction more than turns induced by the auditory information. Our hypothesis is that the strongly degraded sensory information will further reduce the reliability of choices when compared with the arena or other laboratory approaches, even when the differences in signal traits are substantial. Thus, decisions based on small differences between signal parameters may be less likely under realistic natural conditions, or, alternatively, the time necessary for decision making (arriving at a certain source) could be increased relative to the same task in the indoor arena.

The aim of the present study, therefore, was to perform quantitative phonotaxis experiments on female field crickets outdoors, using two-choice decisions based on differences in the male calling song, similar to those performed under laboratory conditions. We varied values of signal traits within the range of variation of the naturally occurring parameter space of populations in carrier frequency, sound pressure level (SPL), and chirp rate. Following the argument made by Bee et al. (2012) in their study on sound pressure level discrimination in tree frogs, we consider that such tests will estimate the "just meaningful difference" (JMD; Nelson and Marler 1990), and not "just noticeable differences" (JNDs) because in behavioural experiments it is possible that the nervous system of receivers may discriminate smaller differences in signal traits under ideal conditions which, however, cannot be used for behavioural discrimination because of noise on the transmission channel. Our results demonstrate that for all three song parameters the JMD between two song models for a significant preference of females was considerably larger in the field compared to previously reported differences in classical laboratory approaches.

\section{Materials and methods}

Study species and phonotaxis in the field

The study was conducted between August and October 2011 and May and September 2012. We used female crickets (Gryllus bimaculatus de Geer) from a population kept at a constant photo cycle of $12 \mathrm{~h}: 12 \mathrm{~h} \mathrm{~L}: \mathrm{D}$ and an ambient 
temperature of $25-30{ }^{\circ} \mathrm{C}$ at the Department of Zoology, University of Graz. Crickets were fed on water gel, fish food, oat flakes and lettuce ad libitum. Last instar females were separated from males to maintain their phonotactic responsiveness. They were kept until the final moult in groups with other females, and isolated acoustically from singing males in a separate room.

We tested the phonotactic behaviour of 97 females 1 week after their final moult in a natural grassland habitat in the vicinity of Graz, Austria. The test ground was totally isolated from traffic noise due to surrounding forest and meadows $\left(47^{\circ} 6^{\prime} 1.2348^{\prime \prime} \mathrm{N} ; 1^{\circ} 27^{\prime} 9.7272^{\prime \prime} \mathrm{E}\right)$. Furthermore, the only other native cricket species in this area, Gryllus campestris, did not sing during the time we performed our experiments, so that masking noise in the frequency band around $5 \mathrm{kHz}$ was largely absent, except for occasional bird song. The height of vegetation on the testing ground was kept between $5 \mathrm{~cm}$ and $10 \mathrm{~cm}$, which was high enough to strongly restrict females in their freedom to walk straight ahead, but nevertheless allowed reliable video tracking from above (see below). To record female phonotaxis, we mounted a custom-made sliding holder onto a steel rope at a height of $2.5 \mathrm{~m}$ above the experimental area, to which a SONY handycam HDRXR155E (Minato, Tokyo, Japan) was attached. The construction allowed moving the video camera over the whole length of the experimental area via cable control, recording the female moving from the release point to the target speaker(s). The display of the camera covered the whole width of the experimental area (given by detours of the females from a straight path to the target), and the magnification was high enough to monitor the position of females within the grass for offline quantitative analysis of their phonotactic approaches, but insufficient to resolve the longitudinal body axis of the insect.

Acoustic stimulation

For playback experiments, we digitally generated models of male calling songs with Cool Edit Pro software (Version 2.0; Syntrillium, Phoenix, AZ, USA) at a sampling rate of $48 \mathrm{k}$-samples/s. The temporal structure of a conspecific chirp was maintained in all song models, with four sound pulses of $23 \mathrm{~ms}$ duration separated by a constant inter pulse interval of $16 \mathrm{~ms}$, resulting in a chirp duration of $140 \mathrm{~ms}$. We modelled variation in male calling songs of natural populations, by varying either the carrier frequency or the chirp rate of song models. Chirps were repeated every $600 \mathrm{~ms}, 462 \mathrm{~ms}$ or $400 \mathrm{~ms}$ to produce chirp rates of 100 , 130 or $150 \mathrm{chirps} / \mathrm{min}$, respectively. In addition, five different carrier frequencies of model songs (4.0, 4.4, 4.7, 4.9 and $5.3 \mathrm{kHz}$ ) were used. $4.9 \mathrm{kHz}$ matched the average best frequency of female receivers, and all other carrier frequencies cover the range of variation of male calling songs in natural populations of G. bimaculatus (Ferreira and Ferguson 2002; Kostarakos et al. 2008).

Each song model was broadcast as an uncompressed WAV-file via an X4-Tech Bogieman IV MP3 player (Braunschweig, Germany) connected to a Kemo Germany M033 amplifier (Langen, Germany) and a full range speaker (Monacor Electronic, SP-626/8, Zwischenwasser, Austria; frequency response $600-18,000 \mathrm{~Hz}$ ). We positioned the speaker $1 \mathrm{~cm}$ from the ground of the experimental area. The SPL of all signals was calibrated using a Rion NL-21 sound level meter and UC-52 $\frac{1}{2} 2$ inch free-field microphone (Tokyo, Japan). Depending on the choice situation of the experiment (see below), signal intensity was adjusted to 85 , 82 or $80 \mathrm{~dB}$ re $20 \mu \mathrm{Pa}$, respectively, at a distance of $50 \mathrm{~cm}$ to the speaker at a height of $1 \mathrm{~cm}$ from the ground, which is about the height of the females approaching the sound source. To characterize the sound field for an approaching female, we also measured the SPL of the signal at distances ranging from 20 to $200 \mathrm{~cm}$ from the speaker in steps of $20 \mathrm{~cm}$. The average SPL of calling songs calibrated to $85 \mathrm{~dB}$ at $50 \mathrm{~cm}$ off the speaker was about $62 \mathrm{~dB}$ SPL at a distance of $200 \mathrm{~cm}$ (Fig. 1). This is about $20 \mathrm{~dB}$ above the absolute hearing threshold of G. bimaculatus (Horseman and Huber 1994; Kostarakos et al. 2008).

Experimental procedure

In preliminary no-choice tests on the experimental ground, we observed phonotactic approaches of females towards the speaker broadcasting a standard model song of $4.9 \mathrm{kHz}$ and $85 \mathrm{~dB}$ SPL, when released at a distance of up to $5 \mathrm{~m}$. However, to quantify and compare with similar approaches in a laboratory arena, we defined a release point at a shorter

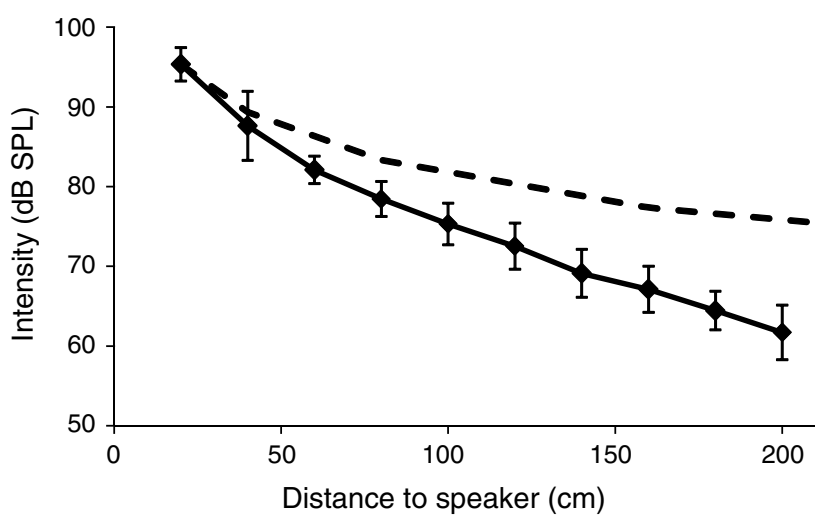

Fig. 1 Attenuation over distance at the testing area for a signal with a carrier frequency of $4.9 \mathrm{kHz}$ calibrated to $85 \mathrm{~dB}$ re $20 \mu \mathrm{Pa}$ at a distance of $50 \mathrm{~cm}$ from the speaker (mean \pm standard deviation). Dashed line represents spreading loss of $6 \mathrm{~dB}$ per doubling of distance 
distance of $200 \mathrm{~cm}$ from the speakers. In two-choice tests, speakers were separated by $180 \mathrm{~cm}$ from each other, so the two sound sources and the release point confined an angle of about $55^{\circ}$.

Female crickets were transported to the testing site separated individually in plastic boxes, where they were left close to the release point for $30 \mathrm{~min}$ to adapt to the new conditions. Ten minutes before testing, females were exposed to low SPLs of calling songs, which they could perceive but not localize due to the closed plastic walls of the boxes. For the start of experiments, females were released by carefully overturning the uncovered plastic boxes. We defined the start of the phonotactic approach as the very moment when the female stepped out of her cage and was free to move. The end of the approach was considered when the female reached a hemicycle with a radius of $10 \mathrm{~cm}$ around the centre of a speaker, although $90 \%$ of females directly encountered the membrane of the speaker. The experimenter was positioned $5 \mathrm{~m}$ away from the test area and controlled the match of the camera's display detail and the cricket's position via a SONY video walkman GV-D900E PAL (Tokyo, Japan) connected to the video camera sliding device above the walking female.

Prior to the start of a new run, we measured the air temperature near the position of the cricket. After a female finished a trial she was given a pause of at least $20 \mathrm{~min}$, before we tested her again in another choice situation. When a female participated in all choice situations or when she did not show phonotactic behaviour on three consecutive days, she was returned to the lab population. Approximately $5 \%$ of all tested crickets did not show phonotaxis at all.

Females were confronted with a two-choice situation with a standard song model (carrier frequency $4.9 \mathrm{kHz}$, chirp rate 150 chirps/min, $85 \mathrm{~dB}$ SPL) and a simultaneously broadcast alternative model song, varying either in carrier frequency, chirp rate, or SPL. All choices are summarized in Table 1. The sequence of choices was randomized. We also performed two-choice tests where a potential preference for higher SPL was traded against a preference for higher chirp rate. Thus, females had a choice between a simultaneous broadcast of a model song with high amplitude of $85 \mathrm{~dB}$ SPL, but a low chirp rate of 100 chirps/min and a model song of $80 \mathrm{~dB}$ SPL, but with a chirp rate of $150 \mathrm{chirps} / \mathrm{min}$. In two-choice experiments, the temporal pattern of the alternative stimulus was presented via the opposite speaker in a time-shifted fashion, so that females were exposed to alternating chirps on the left and right speaker, respectively, broadcasting one chirp at one speaker during the inter-chirp interval of the alternative stimulus.

To avoid a potential side bias in the outdoor setup or asymmetries in the sound propagation due to physical conditions of the grassland transmission channel, we switched the two alternative stimuli between the speakers and retested the females for the same choice condition. Importantly, we also randomized the position of the starting point on the test field within a range of $6 \mathrm{~m}$ day by day. Thus, females did not experience exactly the same transmission channel, such as plaques of grass bundles, in a new trial.

\section{Data analysis}

Video files of phonotactic tracks were converted from .mts type to avi using Any Video Converter (version 3.3.3), and two frames per second of the recordings were cut out for later analysis using VirtualDub (version 1.9.11). These frames were imported in ImageJ 1.44p (Rasband 1997-2011), which allowed a precise frame by frame identification of $x$ - and $y$-positions of a female via MTrackJ plugin (Meijering et al. 2012), so that complete phonotactic approaches could be reproduced in Microsoft Excel. For the analysis of decisions of females, we carried out mixed-model analysis with language $\mathrm{R}$ (R Core Team 2013) and the lme4 package (Bates et al. 2012), which takes into account the non-independent

Table 1 Summarised results of all two-choice experiments

\begin{tabular}{|c|c|c|c|c|c|c|c|}
\hline \multicolumn{3}{|c|}{ Parameters of song model 1} & \multicolumn{3}{|c|}{ Parameters of song model 2} & \multirow{2}{*}{$\begin{array}{l}P \text { value for two-choice } \\
\text { situation (GLMM } \\
\text { binomial model) }\end{array}$} & \multirow{2}{*}{$\begin{array}{l}\text { Number of approaches to } \\
\text { song model } 1 \text { (left) and } \\
\text { song model } 2 \text { (right) }\end{array}$} \\
\hline $\begin{array}{l}\text { SPL }(d B \text { re } \\
20 \mu \mathrm{Pa})\end{array}$ & $\begin{array}{l}\text { Chirp rate } \\
\text { (chirps/min) }\end{array}$ & $\begin{array}{l}\mathrm{CF} \\
(\mathrm{kHz})\end{array}$ & $\begin{array}{l}\text { SPL (dB re } \\
20 \mu \mathrm{Pa})\end{array}$ & $\begin{array}{l}\text { Chirp rate } \\
\text { (chirps/min) }\end{array}$ & $\begin{array}{l}\mathrm{CF} \\
(\mathrm{kHz})\end{array}$ & & \\
\hline 85 & 150 & 4.9 & 82 & 150 & 4.9 & 0.287 & $32(57.1 \%)$ vs. $24(42.9 \%)$ \\
\hline 85 & 150 & 4.9 & 80 & 150 & 4.9 & $<0.001$ & $45(81.8 \%)$ vs. $10(18.2 \%)$ \\
\hline 85 & 150 & 4.9 & 85 & 130 & 4.9 & 0.549 & $54(54 \%)$ vs. $46(46 \%)$ \\
\hline 85 & 150 & 4.9 & 85 & 100 & 4.9 & $<0.01$ & $39(70.9 \%)$ vs. $16(29.1 \%)$ \\
\hline 85 & 150 & 4.9 & 85 & 150 & 5.3 & 0.0613 & $39(61.9 \%)$ vs. $24(38.1 \%)$ \\
\hline 85 & 150 & 4.9 & 85 & 150 & 4.7 & 0.138 & $9(50 \%)$ vs. $9(50 \%)$ \\
\hline 85 & 150 & 4.9 & 85 & 150 & 4.4 & $<0.001$ & $45(72.6 \%)$ vs. $17(27.4 \%)$ \\
\hline 85 & 150 & 4.9 & 85 & 150 & 4.0 & $<0.001$ & $30(83.3 \%)$ vs. $6(16.6 \%)$ \\
\hline 85 & 100 & 4.9 & 80 & 150 & 4.9 & 0.32 & $21(58.3 \%)$ vs. $15(41.7 \%)$ \\
\hline
\end{tabular}

Values in bold indicate parameters which differ between song models 
nature of repeated measurements of same individuals. Since some females lost their motivation for phonotaxis after several trials, they were tested only once in a particular choice situation, whereas the majority of females were tested twice when stimuli were switched between speakers. Thus, it was necessary to fit a generalized linear mixed model (GLMM) including the animal identity as variable. For a comparison of the absolute detour length of phonotactic tracks and females' walking speed obtained in different choice situations, we used for each individual the mean value of observations from each stimulus combination. We computed Kruskal-Wallis ANOVA on ranks, if a Shapiro-Wilk test for normality failed. To isolate the groups which differ from the others, post hoc all pairwise multiple comparison procedure after Dunn's Method was performed. This analysis was carried out using SigmaPlot statistical software (Version 12.0; Systat Software Inc., Chicago, IL, USA).

\section{Results}

Figure 2 shows examples of ten phonotactic tracks for the no-choice situation and for four two-choice situations, respectively.
No-choice trials

In 44 no-choice trials, all female crickets approached the target speaker broadcasting the standard song model. For the shortest possible straight path of $200 \mathrm{~cm}$, females walked an average distance of $306 \mathrm{~cm}$ with a high variation in the detour ranging from 34 to $264 \mathrm{~cm}$ (Fig. 2a). The average time taken for covering this distance was $107.3 \mathrm{~s}$, resulting in an average walking speed of $3.39 \mathrm{~cm} / \mathrm{s}$. Temperature during these approaches covered a range from 18 to $26.6{ }^{\circ} \mathrm{C}$, and with higher temperatures females approached the target faster (Fig. 3).

Two-choice trials with differences in SPL

In a choice between two song alternatives solely differing in SPL, female crickets did not significantly approach the louder sound source, when the difference in SPL was only $3 \mathrm{~dB}$ (GLMM: $N=56, P=0.287$ ). Females approached the louder (85 dB SPL) or softer (82 dB SPL) standard model song in $32(57.1 \%)$ and $24(42.9 \%)$ trials, respectively (Fig. 2e). An increase in the amplitude difference to $5 \mathrm{~dB}$ ( 85 vs. $80 \mathrm{~dB}$ SPL) between the two alternatives resulted in a significant preference for the louder calling
Fig. 2 Cricket phonotaxis in the field under different stimulus situations. For each situation (no-choice (a); high (b) and low (c) difference in carrier frequency; high (d) and low (e) difference in song amplitude) ten examples of phonotactic tracks are provided. Notice that the quality of phonotactic tracks does not differ between twochoice and no-choice situations; for details see text. Phonotactic tracks in red indicate final decisions for the standard signal and tracks in blue indicate final decisions for the alternative song model a

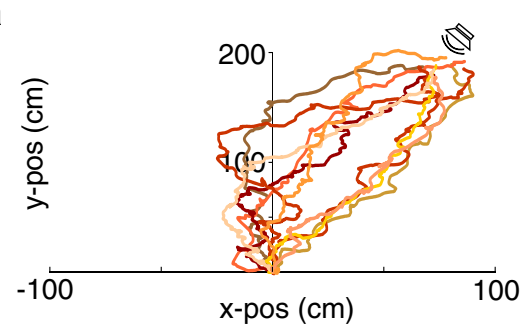

b

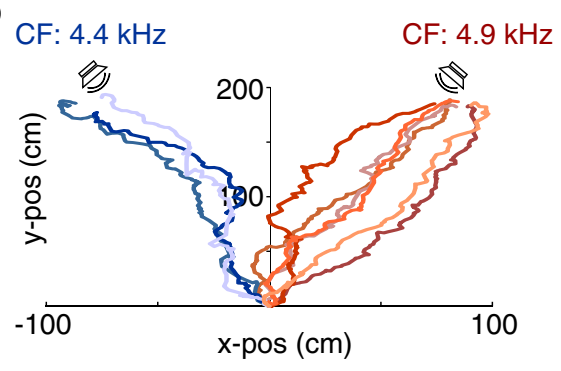

C

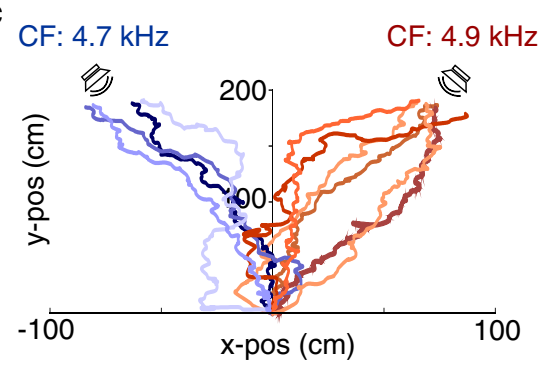

d

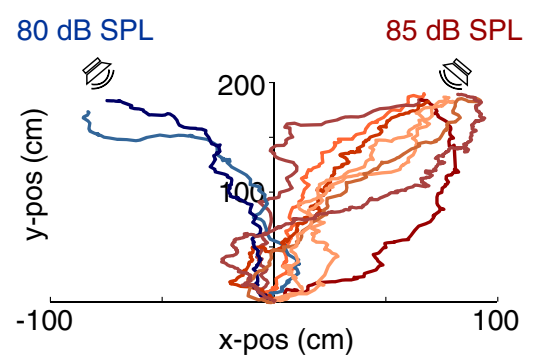

e

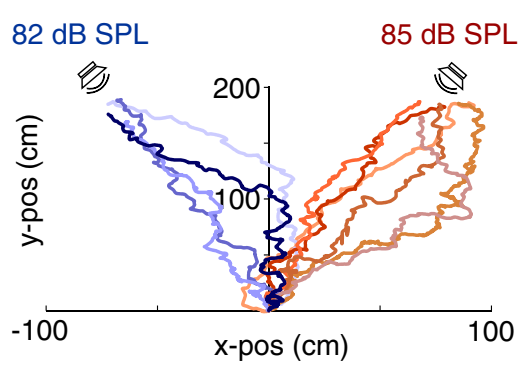




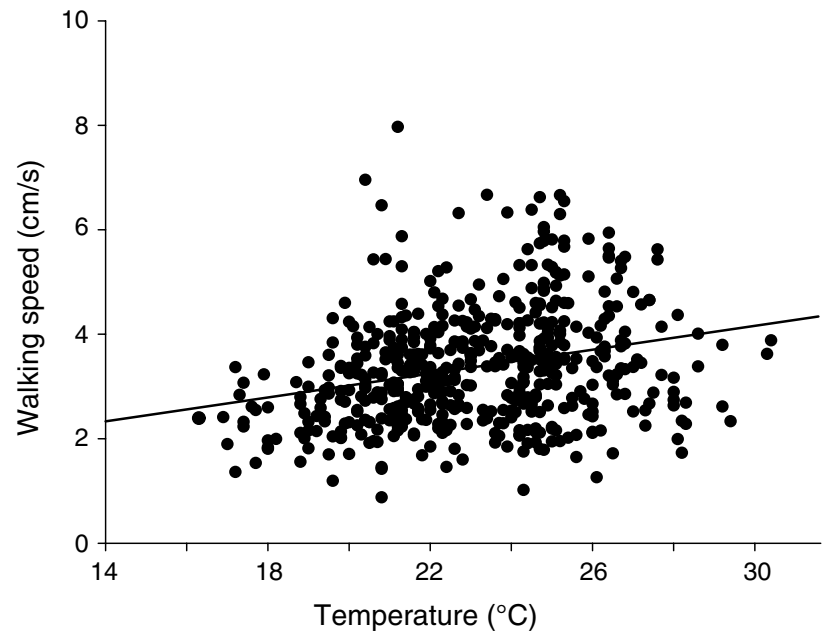

Fig. 3 Correlation between walking speed of female G. bimaculatus in their natural habitat and ambient temperature at the starting point

song (GLMM: $N=55, P<0.001)$, with $45(81.8 \%)$ and $10(18.2 \%)$ females approaching the louder or softer sound source, respectively (Fig. 2d).

Two-choice trials with differences in chirp rate

Similar results were obtained when testing female preference for chirp rate differences. In a choice between model songs differing in chirp rate by only 20 chirps/min ( 150 vs. $130 \mathrm{chirps} / \mathrm{min}$ ) there was no significant preference for the higher chirp rate (GLMM: $N=100, P=0.549$ ), and females approached the higher or lower rate almost equally often (54 and 46 trials, respectively). When the rate difference was increased to 50 chirps/min (150 vs. 100 chirps/ $\min$ ), the higher chirp rate was preferred in 39 out of 55 trials $(70.9 \%)($ GLMM: $N=55, P<0.01)$.

We also traded the two observed preferences for a difference in SPL of $5 \mathrm{~dB}$ with a preference for a difference in chirp rate by 50 chirps/min. We simultaneously presented a model song of high amplitude and low chirp rate (85 dB SPL, 100 chirps/min) and one of low amplitude and high chirp rate $(80 \mathrm{~dB}$ SPL, 150 chirps/min) from either of both speakers. There was no significant preference for either calling song model; females approached the high amplitude model in $21(58.3 \%)$ trials and the high chirp rate model in $15(41.7 \%)$ trials (GLMM: $N=36, P=0.32$ ).

Two-choice trials with differences in carrier frequency

Four different carrier frequencies representing the range of variation in natural populations were tested against a standard carrier frequency of $4.9 \mathrm{kHz}$, which represents the mean best frequency of hearing in G. bimaculatus
(Kostarakos et al. 2009). In such two-choice tests, females did not discriminate between the standard carrier frequency of $4.9 \mathrm{kHz}$ and model songs of $4.7 \mathrm{kHz}$ (Fig. 2c; GLMM: $N=18, P=0.138)$. Even a difference in carrier frequency of $400 \mathrm{~Hz}$ ( 4.9 vs. $5.3 \mathrm{kHz}$ ) was not sufficient for a significant preference (GLMM: $N=63, P=0.0613$ ). However, a significant preference was observed in a choice between the standard model at $4.9 \mathrm{kHz}$ and alternative model at either $4.4 \mathrm{kHz}$ or $4.0 \mathrm{kHz}$. In $45(72.6 \%)$ out of 62 trials, females did chose $4.9 \mathrm{kHz}$ over the alternative at $4.4 \mathrm{kHz}$ (Fig. 2b; GLMM: $N=62, P<0.001)$, in $30(83.3 \%)$ out of 36 trials they preferred the standard over a carrier frequency of $4.0 \mathrm{kHz}$ (GLMM: $N=36, P<0.001$ ).

Comparison of no-choice trials with two-choice trials

We calculated the lengths of phonotactic detours for each choice situation and compared them with the detour values obtained in no-choice experiments. There was a statistical difference between the groups (Shapiro-Wilk test: $P<0.05$; Kruskal-Wallis test: $H 9=24.144, P=0.004$ ), but a post hoc multiple comparison procedure after Dunn's method revealed that the length of detours in no-choice trials did not differ from detour values obtained in any two-choice situation. Instead, there was a difference in the groups with the highest median (trading experiment; median $121.6 \mathrm{~cm}$ ) and lowest median (frequency test with an alternative stimulus carrier frequency of $4.4 \mathrm{kHz}$; median $76.8 \mathrm{~cm}$ ) for detour length.

The analysis of walking speed revealed similar results. The walking speed of females in a no-choice situation did not differ from their speed in two-choice experiments, but again there was a difference in the groups with the lowest median (trading experiment; median $2.8 \mathrm{~cm} / \mathrm{s}$ ) and highest median (frequency test with an alternative stimulus carrier frequency of $4.4 \mathrm{kHz}$; median $3.7 \mathrm{~cm} / \mathrm{s}$ ) for walking speed (Shapiro-Wilk test: $P<0.05$; Kruskal-Wallis test: $H 9=19.399, P=0.022$; post hoc all pairwise multiple comparison procedure after Dunn's method).

To quantify the dependence of walking speed on temperature we performed a correlation analysis including every single data point, ignoring multiple testing of an individual. We found a linear correlation between walking speed (Spearman rank correlation: $r_{\mathrm{s}}=0.281, N=521$, $P<0.001)$ and temperature ranging from 16.3 to $30.4{ }^{\circ} \mathrm{C}$ (Fig. 3); however, the dependency of walking speed from ambient temperature is rather weak. Females did not show any phonotactic behaviour below a temperature of $16^{\circ} \mathrm{C}$.

\section{Discussion}

Numerous studies in the past using different behavioural paradigms provided evidence that the SPL, carrier 
frequency, or chirp rate influences female choice of crickets in controlled lab conditions (for review see Gerhardt and Huber 2002). A general result was that small differences in these parameters were sufficient to create differences in phonotactic scores to song models. However, female mate choice through phonotaxis has only rarely been observed and quantified in its natural environment. The aim of the present study was, therefore, to describe cricket phonotaxis outdoors and to compare the behavioural data with those obtained under the rather different lab conditions. Although our data confirm the general preference of females in two-choice tests for higher SPL and chirp rate, and for a medium carrier frequency of $4.9 \mathrm{kHz}$, the field data demonstrated that the necessary differences between two sound stimuli for a significant choice were considerably larger in the natural environment.

\section{Differences in SPL}

In crickets and katydids, differences in the SPL of signals are of relevance both for the outcome of male-male interactions (Dadour and Bailey 1985; Latimer and Schatral 1986) and for preferences exhibited by females in a two-choice situation (Shuvalov and Popov 1973; Gwynne 1982; Doherty 1985; Latimer and Sippel 1987; Bailey and Yeoh 1988; Weber and Thorson 1988; Bailey et al. 1990). One source of evidence for the minimum binaural cues involved for orientation stems from female choice experiments with two sound sources presented from opposite directions (partly summarized by Forrest 1994; Römer et al. 1998; see also Gerhardt and Huber 2002 and literature therein). Depending on species and experimental design, insects prefer one source over the other when the difference is $1-3 \mathrm{~dB}$. The highest sensitivity for differences in SPL was reported when acoustic stimulation was precisely controlled in experiments using dichotic ear stimulation techniques: both grasshoppers and katydids appear able to detect interaural intensity differences (IIDs) as small as $0.5-1 \mathrm{~dB}$ that reliably elicit a turn to the louder side (von Helversen and Rheinlaender 1988; Rheinlaender et al. 2005). Similarly, when tested on a fast trackball system, female G. bimaculatus exhibit a high sensitivity towards interaural differences in sound intensity and orient towards the louder of two sound sources at IIDs of $1 \mathrm{~dB}$ and less (Hedwig and Poulet 2005; Schöneich and Hedwig 2010). Altogether, such experiments reveal the best sensory capacity and resolution for intensity differences of the auditory system available to the insects. However, in two-choice arena trials with female G. bimaculatus using an identical stimulus protocol as in the outdoor experiments such small differences in SPL of otherwise identical stimuli have been insufficient for a clear preference for the higher SPL; rather a minimum intensity difference of $3 \mathrm{~dB}$ was required for a significant choice of the louder song model
(Brunnhofer 2011; SH and HR, unpublished results). However, as the outdoor results show, only an increase in the amplitude difference to $5 \mathrm{~dB}$ between the two alternatives resulted in a significant preference for the louder calling song. Despite this significance it is worth mentioning that even in 10 (out of 55) trials females oriented towards, and approached the speaker broadcasting the $5 \mathrm{~dB}$ softer song. Thus, for a comparison of results some of the observed differences on the trackball and in dichotic stimulation experiments on the one hand, and in arena experiments on the other, are due to differences between open- and closed-loop conditions, whereas the further increase in the necessary differences outdoors are due to the noisy conditions for sound transmission.

The fact that females require differences in SPL between two song models as large as $5 \mathrm{~dB}$ for selective phonotaxis may result from the problems associated with sound propagation parallel to the ground, often referred to as "the forbidden mode of propagation" (Piercy et al. 1977). Indeed, in a neurophysiological study at the same site where our behavioural experiments were performed Kostarakos and Römer (2010) reported strong fluctuations in the intensity gradient of a sound signal over distances between 1 and $16 \mathrm{~m}$. Over the relatively short phonotactic distance of $2 \mathrm{~m}$ in the present study such fluctuations appear to be much smaller, although in the summary plot of data from 56 experiments these fluctuations in the intensity gradient will disappear (Fig. 1). It is quite possible though that over larger distances with more pronounced irregularities in the intensity gradient even a $5 \mathrm{~dB}$ difference between song alternatives may be insufficient for a significant preference. However, Kostarakos and Römer (2010) also reported strong and irregular variations in directional information on the transmission channel, sometimes providing wrong directional information to the CNS concerning the azimuthal direction of a single sound source, although under identical stimulus configurations in the undistorted laboratory large and correct differences (i.e., the ipsilateral side firing more strongly) have been observed (Kostarakos et al. 2009). Thus, whatever the reason for such discontinuities, a female in an outdoor two-choice experiment may eventually experience either "silent spots" for one or both signals or locations where binaural information regarding the more intense of two stimuli is distorted. This would explain that differences in SPL between two stimuli need to be larger under natural conditions compared to arena or trackball trials. These large differences are even more surprising, since for crickets and katydids a "selective attention" mechanism has been described with the potential to neurally increase the difference in the representation of two stimuli differing by only a few decibels (Pollack 1988; Römer and Krusch 2000). However, given the unpredictable nature of the intensity and directionality fluctuations, the "selective 
attention" would provide no mechanism to compensate these effects of the transmission channel, since it would operate on SPL differences without discrimination whether the differences represent "true" or "false" information on signal differences.

A further reason for differences concerning mate choice decisions based on signal SPL between lab and outdoor experiments is the physical nature of the grassland in which females have to move and orient. Even if females perceive reliable sensory information about the location of the more intense of two stimuli, they might be forced by dense patches of grass or larger obstacles on the ground to move into another, wrong direction. If such misdirected orientation sequences occur repeatedly, a female might deviate from the correct direction up to a point where she is closer to the less intense source, so that distance effects cancel the original SPL differences at the starting point. Even if she eventually arrives at the correct, more intense signal her phonotactic path would be longer. Support for this interpretation provides the calculation of the deviation of the phonotactic approach from the ideal (straight) line towards the speaker. When compared with the same measure obtained from approaches in the arena under similar choice conditions (Brunnhofer 2011), this deviation is significantly reduced under arena conditions (relative detour values of 0.481 for outdoor experiments vs. 0.173 for arena experiments; Mann-Whitney rank sum test: $T=964.000$, $N=24,29, P \leq 0.001$; see Fig. 4).

One of the most obvious reasons for the observed differences in laboratory and field experiments may be background noise and its masking effect for detection of smaller differences in signals (Brumm and Slabbekoorn 2005;

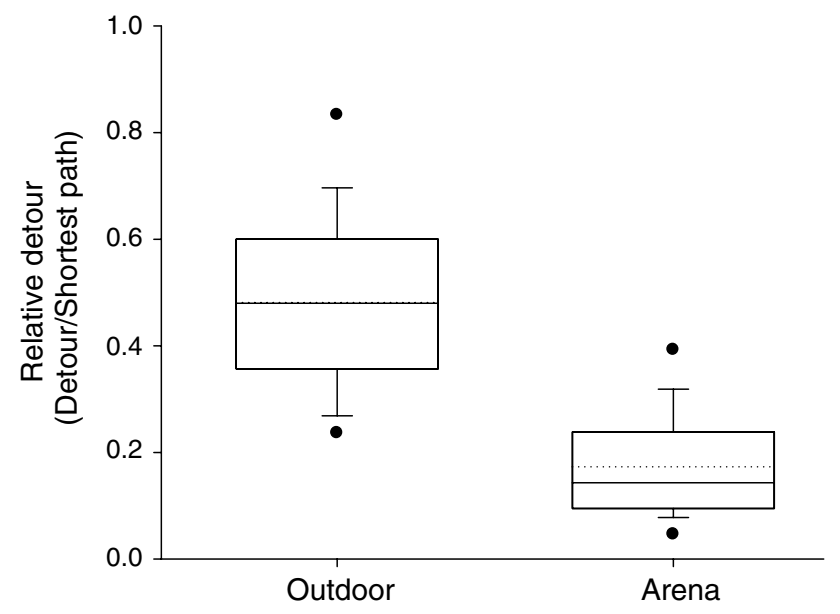

Fig. 4 Comparison of detour lengths of phonotactic tracks recorded in outdoor trials and arena trials. In both paradigms two song alternatives only differed in amplitude by $3 \mathrm{~dB}$. Females covered significant longer detours in outdoor experiments (Mann-Whitney rank sum test: $P \leq 0.001)$
Römer 2013). However, the present study area was totally isolated from traffic noise due to surrounding forest and meadows, and experiments were conducted during a time when the only other native cricket species in this area, $G$. campestris, did not sing. Thus, masking noise in the frequency band around $5 \mathrm{kHz}$ was largely absent, except for occasional bird song (see also Kostarakos and Römer 2010).

Finally, one reason for the differences between results obtained outdoors and in the laboratory may be the variation in temperature, which ranged between 16.3 and $30.4{ }^{\circ} \mathrm{C}$ in our study compared to arena experiments at $21^{\circ} \mathrm{C}$ reported by Brunnhofer (2011). Whereas the increase of walking speed with increasing temperature (Fig. 3) is typical for poikilothermic animals, the effect of temperature or temperature gradients for sound transmission and amplitude fluctuations is unclear (Wiley and Richards 1978). van Staaden and Römer (1997) demonstrated that temperature gradients in the order of $4.5^{\circ} \mathrm{C}$ over $10 \mathrm{~m}$ may strongly increase the active range of the call of a male bladder grasshopper, and much stronger temperature gradients as a result of sun radiation (about $10{ }^{\circ} \mathrm{C}$ over a height of only $30 \mathrm{~cm}$ ) may occur in the grassland habitat of a field cricket (Römer 2001). Whether such gradients play a role for sound transmission and perception for field crickets needs to be investigated.

\section{Differences in carrier frequency}

When tested with reasonable small increments of frequency differences spanning the natural range of variation in male cricket calling songs, most studies reported stabilizing preferences for the carrier frequency of the call (Ulagaraj and Walker 1975; Thorson et al. 1982; Stout et al. 1983; Shaw and Herlihy 2000; Kostarakos et al. 2008; but see Verburgt and Ferguson 2010 below). Such preferences can usually be explained by the frequency tuning of the peripheral auditory system. In $G$. bimaculatus, the female preference for carrier frequency correlates highly with the tuning of a sensory interneuron (AN1; Kostarakos et al. 2008), known for its importance in cricket phonotaxis (Schildberger and Hörner 1988; Atkins et al. 1992). The tuning of AN1 to a best frequency of $4.9 \mathrm{kHz}$ and the roll-off towards lower and higher frequencies represent a "hard-wired" preference function in G. bimaculatus. According to the average tuning of female receivers, frequency differences between two calling songs can be translated into differences in the perceived sound pressure level (Kostarakos et al. 2009). As shown in their study, a frequency difference of $200 \mathrm{~Hz}(4.9$ vs. $4.7 \mathrm{kHz}$ ) equals an intensity difference of $2.6 \mathrm{~dB}$, and a difference of $400 \mathrm{~Hz}$ ( 4.9 vs. $5.3 \mathrm{kHz}$ ) is equivalent to an intensity difference of $3.75 \mathrm{~dB}$. In both of these choice trials, our outdoor results revealed no significant preference 
for the signal at the best frequency of hearing $(4.9 \mathrm{kHz})$, which is consistent with the results obtained with signal differences in SPL, where a minimum difference of $5 \mathrm{~dB}$ was necessary for selective female phonotaxis. Again, the significant preferences observed in a choice for the standard model at $4.9 \mathrm{kHz}$ versus alternatives differing by $500 \mathrm{~Hz}$ and $900 \mathrm{~Hz}$ (4.4 or $4.0 \mathrm{kHz}$, respectively) result in perceived level differences of 9.6 and $23.7 \mathrm{~dB}$, clearly well above the level difference of $5 \mathrm{~dB}$.

Preferences of females for the carrier frequency of the male call are often examined in the context of preferences for indicators of body size, due to the negative correlation of male size and carrier frequency as a result of sound production, where larger resonators produce lower frequencies (for review see Brown 1999; Gerhardt and Huber 2002). However, whereas some studies confirmed female preferences for lower than average carrier frequency in crickets (Simmons and Ritchie 1996; Brown et al. 1996; Scheuber et al. 2004), others do either find no preference for carrier frequency in the same species (Deb et al. 2012), or even a preference for higher over lower frequencies (Kostarakos et al. 2008; the present study). The shape and steepness of the peripheral tuning also determine the sensitivity to small variations in male carrier frequency: behavioural tuning curves in the field crickets Teleogryllus commodus and $T$. oceanicus fall off more sharply at low frequencies compared to high frequencies (Hennig and Weber 1997). Notably, in their study on Oecanthus henryi, Deb et al. (2012) carefully examined these preferences at two SPL values close to hearing threshold and well above threshold, as well as with increments of frequency differences spanning the large range of frequencies to which the system is tuned (2.5-4.5 kHz; Mhatre et al. 2011). Despite the lack of female preference for this call character which is negatively correlated with body size, females nevertheless demonstrated a preference for larger males in several parameters of their mating behaviour. Thus, the finding that larger males have a mating advantage in the field (Simmons 1986) may not be attributed to choice based on acoustic signal parameters, but on close range cues (Thomas and Simmons 2009; Simmons et al. 2013).

Whereas our results on selective phonotaxis in the field are consistent with earlier studies obtained in the lab demonstrating stabilizing selection for a carrier frequency which corresponds to the best frequency of hearing, Verburgt and Ferguson (2010) argued for the same species that females are unable in detecting differences in song carrier frequency of males. However, their results are confounded by the fact that one cannot judge the discriminatory potential of receivers from preference functions obtained in no-choice trials, since females will track a male calling song for a large range of carrier frequency, given the SPL is well above the behavioural threshold for phonotaxis.
Indeed, no-choice trials for carrier frequencies of 4.4, 4.9 and $5.2 \mathrm{kHz}$ did not reveal any significant difference in the absolute amount of lateral steering on a trackball system (Hirtenlehner et al. 2013). In a choice situation, however, the relative intensities of the two signals become highly relevant as a result of the underlying tuning of neuronal elements (Kostarakos et al. 2008). Brown et al. (1996) also found a preference for carrier frequency in female $O$. nigricornis only when they had the opportunity to compare calls broadcast simultaneously.

\section{Differences in chirp rate}

Numerous species of insects and anurans demonstrate female preferences for higher call or chirp rates over lower ones, a parameter which is most variable among individual and populations (Ryan and Keddy-Hector 1992; review in Gerhardt and Huber 2002). Assessing the sensory information for such a dynamic song character poses the problem that the actual value of a difference between two or more signals accumulates only slowly over time. The smaller the difference in chirp rate, the longer it should take for evaluating this information. This creates a dilemma known as the speedaccuracy trade-off (Wickelgren 1977; Chittka et al. 2009). Indeed, under open-loop conditions on a trackball system female $G$. bimaculatus significantly preferred the higher chirp rate when the minimum difference was only 20 chirps/ min, and the lateral steering into the direction of the higher rate reached a predefined threshold earlier with higher rate differences (Trobe et al. 2011). We are unaware of any field studies addressing mate choice for this dynamic property of the calling song alone, but under field conditions, this difference was not sufficient for a preference, and female crickets required a chirp rate difference of 50 chirps/min (150 vs. 100) for a reliable decision for higher rates. During the average time needed by a female over the phonotactic path towards the preferred speaker $(84.6 \mathrm{~s})$ she experienced about 70 chirps more through the signal with the higher chirp rate. Although such large differences between male calling songs may occur, they nevertheless present extremes in the distribution, and may thus not occur very frequently.

\section{Conclusion}

Our data on acoustic preferences of female crickets examined under conditions of natural grassland have shown that the small differences in signal parameters which are sufficient in two-choice trials under various laboratory settings do not provide reliable information outdoors. Similar to the discussion about the importance of fluctuating asymmetry, where many of the reported preferences for symmetrical traits have been obtained with trait values very rarely 
experienced in nature (Swaddle 1999), the present finding has important consequences when considering the strength of sexual selection through female choice. However, we are aware of the problem that a direct comparison between results of the numerous studies on acoustic insects should be treated with caution. They differ in many aspects, such as in their open or closed-loop condition, whether female preferences are tested in no-choice or choice trials, whether the choice was simultaneous or sequential, or whether the choice was based on single or multiple sound sources. A further difference relates to the use of multisensory, in particular visual, information, since our outdoor experiments were performed during the day, and females could potentially use visual landmarks in combination with acoustic directional information, whereas lab experiments are usually conducted in the dark. Moreover, in arena trials the distance covered by females from the starting point to the target was often less than $50 \mathrm{~cm}$ (Mhatre and Balakrishnan 2007, 2008), giving the female less freedom to redirect her path because intensity effects through distance become most important for the decision. Studies also vary considerably in their spatial arrangement of the sound sources, placed either from opposite ends of the arena (separation $180^{\circ}$ ) or values between 50 and $90^{\circ}$, with consequences for the amount of directional information for the alternative stimuli.

Acknowledgments We thank Birgit Rönfeld for processing phonotactic video frames; Helmut Lackner and Manfred Löcker for support in the field, and Kristina Sefc for support in statistical analysis. Research was funded by the Austrian Science Fund (FWF I1054-B25). We thank two anonymous reviewers for their helpful comments which improved the manuscript. The performed experiments comply with the current laws of Austria.

Open Access This article is distributed under the terms of the Creative Commons Attribution License which permits any use, distribution, and reproduction in any medium, provided the original author(s) and the source are credited.

\section{References}

Alexander RD (1962) Evolutionary change in cricket acoustical communication. Evolution 16:443-467. doi:10.2307/2406178

Andersson MB (1994) Sexual selection. Princeton University Press, Princeton

Atkins G, Henley J, Handysides R, Stout J (1992) Evaluation of the behavioral roles of ascending auditory interneurons in calling song phonotaxis by the female cricket (Acheta domesticus). J Comp Physiol A. doi:10.1007/BF00191425

Bailey WJ, Yeoh PB (1988) Female phonotaxis and frequency discrimination in the bushcricket Requena verticalis. Physiol Entomol 13:363-372. doi:10.1111/j.1365-3032.1988.tb01118.x

Bailey WJ, Cunningham RJ, Lebel L (1990) Song power, spectral distribution and female phonotaxis in the bushcricket Requena verticalis (Tettigoniidae: Orthoptera): active female choice or passive attraction. Anim Behav 40:33-42. doi:10.1016/ S0003-3472(05)80663-3
Bates D, Maechler M, Bolker B (2012) lme4: Linear mixed-effects models using $\mathrm{S} 4$ classes

Bee MA, Vélez A, Forester JD (2012) Sound level discrimination by gray treefrogs in the presence and absence of chorus-shaped noise. J Acoust Soc Am 131:4188-4195. doi:10.1121/1.3699271

Bentsen CL, Hunt J, Jennions MD, Brooks R (2006) Complex multivariate sexual selection on male acoustic signaling in a wild population of Teleogryllus commodus. Am Nat 167:E102-E116. doi:10.1086/an.2006.167.issue-4

Bradbury JW, Vehrencamp SL (2011) Principles of animal communication. Sinauer Associates, Sunderland, second edition

Brooks R, Hunt J, Blows MW et al (2005) Experimental evidence for multivariate stabilizing sexual selection. Evolution 59:871-880

Brown WD (1999) Mate choice in tree crickets and their kin. Annu Rev Entomol 44:371-396. doi:10.1146/annurev.ento.44.1.371

Brown WD, Wideman J, Andrade MCB et al (1996) Female choice for an indicator of male size in the song of the Black-Horned Tree Cricket, Oecanthus nigricornis (Orthoptera: Gryllidae: Oecanthinae). Evolution 50:2400-2411. doi:10.2307/2410708

Brumm H, Slabbekoorn H (2005) Acoustic communication in noise. Adv Study Behav. Academic Press, London, pp 151-209

Brunnhofer MH (2011) Choice of Gryllus bimaculatus females for male calling song and signal complexity in arena trials: behavior and neurophysiology. Master thesis. Karl-Franzens University, Graz

Chittka L, Skorupski P, Raine NE (2009) Speed-accuracy tradeoffs in animal decision making. Trends Ecol Evol 24:400-407. doi:10.1016/j.tree.2009.02.010

Dadour IR, Bailey WJ (1985) Male agonistic behaviour of the bushcricket Mygalopsis marki Bailey in response to conspecific song (Orthoptera: Tettigoniidae). Z Tierpsychol 70:320-330. doi:10.1111/j.1439-0310.1985.tb00521.x

Deb R, Bhattacharya M, Balakrishnan R (2012) Females of a tree cricket prefer larger males but not the lower frequency male calls that indicate large body size. Anim Behav 84:137-149. doi:10.1016/j.anbehav.2012.04.020

Doherty JA (1985) Trade-off phenomena in calling song recognition and phonotaxis in the cricket, Gryllus bimaculatus (Orthoptera, Gryllidae). J Comp Physiol A 156:787-801. doi:10.1007 /BF00610831

Dyson ML, Passmore NI, Bishop PJ, Henzi SP (1992) Male behavior and correlates of mating success in a natural population of African painted reed frogs (Hyperolius marmoratus). Herpetologica 48:236-246

Embleton TFW (1996) Tutorial on sound propagation outdoors. J Acoust Soc Am 100:31-48. doi:10.1121/1.415879

Endler JA (1992) Signals, signal conditions, and the direction of evolution. Am Nat 139:S125-S153

Farris HE, Forrest TG, Hoy RR (1998) The effect of ultrasound on the attractiveness of acoustic mating signals. Physiol Entomol 23:322-328. doi:10.1046/j.1365-3032.1998.234093.x

Ferreira M, Ferguson JWH (2002) Geographic variation in the calling song of the field cricket Gryllus bimaculatus (Orthoptera: Gryllidae) and its relevance to mate recognition and mate choice. J Zool 257:163-170. doi:10.1017/S0952836902000766

Forrest TG (1994) From sender to receiver: propagation and environmental effects on acoustic signals. Am Zool 34:644-654. doi:10. 1093/icb/34.6.644

Gerhardt HC (1992) Conducting playback experiments and interpreting their results. In: MacGregor P (ed) Playback and studies of animal communication: problems and prospects. NATO Advanced research workshop. Plenum Press, New York, pp 59-77

Gerhardt HC, Huber F (2002) Acoustic communication in insects and anurans: common problems and diverse solutions. University of Chicago Press, Chicago 
Gwynne DT (1982) Mate selection by female katydids (Orthoptera: Tettigoniidae, Conocephalus nigropleurum). Anim Behav 30:734-738. doi:10.1016/S0003-3472(82)80145-0

Hedwig B (2006) Pulses, patterns and paths: neurobiology of acoustic behaviour in crickets. J Comp Physiol A 192:677-689. doi:10.1007/s00359-006-0115-8

Hedwig B, Poulet JFA (2005) Mechanisms underlying phonotactic steering in the cricket Gryllus bimaculatus revealed with a fast trackball system. J Exp Biol 208:915-927. doi:10.1242/jeb.01452

Hennig RM, Weber T (1997) Filtering of temporal parameters of the calling song by cricket females of two closely related species: a behavioral analysis. J Comp Physiol A 180:621-630. doi: $10.1007 / \mathrm{s} 003590050078$

Hirtenlehner S, Küng S, Kainz F, Römer H (2013) Asymmetry in cricket song: female preference and proximate mechanism of discrimination. J Exp Biol 216:2046-2054. doi:10.1242/jeb.083774

Hirtenlehner S, Römer H, Schmidt AKD (2014) Out of phase: relevance of the medial septum for directional hearing and phonotaxis in the natural habitat of field crickets. J Comp Physiol A 200:139-148. doi:10.1007/s00359-013-0869-8

Horseman G, Huber F (1994) Sound localisation in crickets. J Comp Physiol A 175:399-413. doi:10.1007/BF00199248

Kostarakos K, Römer H (2010) Sound transmission and directional hearing in field crickets: neurophysiological studies outdoors. J Comp Physiol A 196:669-681. doi:10.1007/s00359-010-0557-x

Kostarakos K, Hartbauer M, Römer H (2008) Matched filters, mate choice and the evolution of sexually selected traits. PLoS ONE 3:e3005. doi:10.1371/journal.pone.0003005

Kostarakos K, Hennig MR, Römer H (2009) Two matched filters and the evolution of mating signals in four species of cricket. Front Zool 6:22. doi:10.1186/1742-9994-6-22

Latimer W, Schatral A (1986) Information cues used in male competition by Tettigonia cantans (Orthoptera: Tettigoniidae). Anim Behav 34, Part 1:162-168. doi:10.1016/0003-3472(86)90019-9

Latimer W, Sippel M (1987) Acoustic cues for female choice and male competition in Tettigonia cantans. Anim Behav 35:887900. doi:10.1016/S0003-3472(87)80124-0

Meijering E, Dzyubachyk O, Smal I (2012) Methods for cell and particle tracking. Methods Enzymol 504:183-200. doi:10.1016/ B978-0-12-391857-4.00009-4

Mhatre N, Balakrishnan R (2007) Phonotactic walking paths of field crickets in closed-loop conditions and their simulation using a stochastic model. J Exp Biol 210:3661-3676. doi:10.1242/jeb.003764

Mhatre N, Balakrishnan R (2008) Predicting acoustic orientation in complex real-world environments. J Exp Biol 211:2779-2785. doi:10.1242/jeb.017756

Mhatre N, Bhattacharya M, Robert D, Balakrishnan R (2011) Matching sender and receiver: poikilothermy and frequency tuning in a tree cricket. J Exp Biol 214:2569-2578. doi:10.1242/jeb.057612

Nelson DA, Marler P (1990) The perception of birdsong and an ecological concept of signal space. In: Stebbins WC, Berkley MA (eds) Comp percept. Complex signals, vol 2. Wiley, Oxford, pp 443-478

Otte D (1992) Evolution of cricket songs. J Orthop Res 1:25-49. doi: $10.2307 / 3503559$

Piercy JE, Embleton TFW, Sutherland LC (1977) Review of noise propagation in the atmosphere. J Acoust Soc Am 61:1403-1418. doi:10.1121/1.381455

Pollack G (1988) Selective attention in an insect auditory neuron. J Neurosci 8:2635-2639

Pollack GS (1998) Neural processing of acoustic signals. In: Hoy RR, Popper AN, Fay RR (eds) Comp Hear Insects. Springer, New York, pp 139-196
R Core Team (2013) R: a language and environment for statistical computing Rasband WS (1997-2011) ImageJ. U. S. National Institutes of Health, Bethesda, Maryland. http://rsbweb.nih.gov/ij/

Rheinlaender J, Shen J-X, Römer H (2005) Auditory lateralization in bushcrickets: a new dichotic paradigm. J Comp Physiol A 192:389-397. doi:10.1007/s00359-005-0078-1

Rodríguez-Muñoz R, Bretman A, Slate J et al (2010) Natural and sexual selection in a wild insect population. Science 328:1269-1272. doi:10.1126/science. 1188102

Römer H (1998) The sensory ecology of acoustic communication in Insects. In: Hoy RR, Popper AN, Fay RR (eds) Comp Hear Insects. Springer, New York, pp 63-96

Römer H (2001) Ecological constraints for sound communication: from grasshoppers to elephants. In: Barth FG, Schmid A (eds) Ecol Sens. Springer, New York, pp 59-77

Römer H (2013) Masking by noise in acoustic insects: Problems and solutions. In: Brumm H (ed) Anim. Commun. Noise. Springer, Berlin, pp 33-63. doi:10.1007/978-3-642-41494-7_3

Römer H, Krusch M (2000) A gain-control mechanism for processing of chorus sounds in the afferent auditory pathway of the bushcricket Tettigonia viridissima (Orthoptera; Tettigoniidae). J Comp Physiol A 186:181-191. doi:10.1007/s003590050018

Römer H, Lewald J (1992) High-frequency sound transmission in natural habitats: implications for the evolution of insect acoustic communication. Behav Ecol Sociobiol 29:437-444. doi:10.1007 /BF00170174

Römer H, Spickermann M, Bailey W (1998) Sensory basis for sound intensity discrimination in the bushcricket Requena verticalis (Tettigoniidae, Orthoptera). J Comp Physiol A 182:595-607. doi: $10.1007 / \mathrm{s} 003590050206$

Ryan MJ, Keddy-Hector A (1992) Directional patterns of female mate choice and the role of sensory biases. Am Nat 139:S4-S35

Scheuber H, Jacot A, Brinkhof MWG (2004) Female preference for multiple condition-dependent components of a sexually selected signal. Proc R Soc Lond B Biol Sci 271:2453-2457. doi:10.1098 /rspb2004.2907

Schildberger K, Hörner M (1988) The function of auditory neurons in cricket phonotaxis. J Comp Physiol A 163:621-631. doi:10.1007 /BF00603846

Schöneich S, Hedwig B (2010) Hyperacute directional hearing and phonotactic steering in the cricket (Gryllus bimaculatus deGeer). PLoS ONE 5:e15141. doi:10.1371/journal.pone.0015141

Shaw KL, Herlihy DP (2000) Acoustic preference functions and song variability in the Hawaiian cricket Laupala cerasina. Proc R Soc Lond B Biol Sci 267:577-584. doi:10.1098/rspb2000.1040 2000.1040

Shuvalov VF, Popov AV (1973) Significance of some parameters of the calling songs of male crickets, Gryllus bimaculatus for phonotaxis of females. J Evol Biochem Physiol 9:177-182

Simmons L (1986) Inter-male competition and mating success in the field cricket, Gryllus bimaculatus (de Geer). Anim Behav 34:567-579. doi:10.1016/s0003-3472(86)80126-9

Simmons LW, Ritchie MG (1996) Symmetry in the songs of crickets. Proc R Soc Lond B Biol Sci 263:1305-1311. doi:10.1098/r spb1996.0191

Simmons LW, Thomas ML, Simmons FW, Zuk M (2013) Female preferences for acoustic and olfactory signals during courtship: male crickets send multiple messages. Behav Ecol art036. doi:10.1093/beheco/art036

Singer TL (1998) Roles of hydrocarbons in the recognition systems of insects. Am Zool 38:394-405. doi:10.1093/icb/38.2.394

Stout JF, DeHaan CH, McGhee RW (1983) Attractiveness of the male Acheta domesticus calling song to females. J Comp Physiol A 153:509-521. doi:10.1007/BF00612605 
Swaddle JP (1999) Visual signalling by asymmetry: a review of perceptual processes. Philos Trans R Soc Lond B Biol Sci 354:13831393. doi:10.1098/rstb1999.0486

Thomas ML, Simmons LW (2009) Sexual selection on cuticular hydrocarbons in the Australian field cricket, Teleogryllus oceanicus. BMC Evol Biol 9:162. doi:10.1186/1471-2148-9-162

Thorson J, Weber T, Huber F (1982) Auditory behavior of the cricket. J Comp Physiol A 146:361-378. doi:10.1007/BF00612706

Trobe D, Schuster R, Römer H (2011) Fast and reliable decisions for a dynamic song parameter in field crickets. J Comp Physiol A 197:131-135. doi:10.1007/s00359-010-0589-2

Ulagaraj SM, Walker TJ (1975) Response of flying mole crickets to three parameters of synthetic songs broadcast outdoors. Nature 253:530-532. doi:10.1038/253530a0

van Staaden MJ, Römer H (1997) Sexual signalling in bladder grasshoppers: tactical design for maximizing calling range. J Exp Biol 200:2597-2608

Verburgt L, Ferguson JWH (2010) Mate choice in field crickets: can females acoustically detect male body size? J Ethol 28:141-151. doi:10.1007/s10164-009-0166-8 von Helversen D, Rheinlaender J (1988) Interaural intensity and time discrimination in an unrestraint grasshopper: a tentative behavioural approach. J Comp Physiol A 162:333-340. doi:10.1007 /BF00606121

Wagner WE, Reiser MG (2000) The importance of calling song and courtship song in female mate choice in the variable field cricket. Anim Behav 59:1219-1226. doi:10.1006/anbe1999.1428

Walker TJ, Forrest TG (1989) Mole Cricket Phonotaxis: effects of Intensity of Synthetic Calling Song (Orthoptera: Gryllotalpidae: Scapteriscus acletus). Fla Entomol 72:655-659. doi: $10.2307 / 3495043$

Weber T, Thorson J (1988) Auditory behavior of the cricket. J Comp Physiol A 163:13-22. doi:10.1007/BF00611992

Wickelgren WA (1977) Speed-accuracy tradeoff and information processing dynamics. Acta Psychol (Amst) 41:67-85. doi:10.1016/0001-6918(77)90012-9

Wiley RH, Richards DG (1978) Physical constraints on acoustic communication in the atmosphere: implications for the evolution of animal vocalizations. Behav Ecol Sociobiol 3:69-94. doi:10.100 7/BF00300047 